\title{
A Personalization Effect in Multimedia Learning: Students Learn Better When Words Are in Conversational Style Rather Than Formal Style
}

\author{
Richard E. Mayer, Sherry Fennell, Lindsay Farmer, and Julie Campbell \\ University of California, Santa Barbara
}

\begin{abstract}
Students received a personalized or nonpersonalized version of a narrated animation explaining how the human respiratory system works. The narration for the nonpersonalized version was in formal style, whereas the narration for the personalized version was in conversational style in which "the" was changed to "your" in 12 places. In 3 experiments, students who received the personalized version scored significantly higher on transfer tests but not on retention tests than did students who received the nonpersonalized version. The results are consistent with a cognitive theory of multimedia learning in which personalization causes students to actively process the incoming material.
\end{abstract}

Suppose you are sitting at your computer, exploring a Web site on health information. You click on a link about how the human respiratory system works, and you see a short presentation on the screen. The presentation consists of an animation depicting the processes of inhaling air into the lungs, exchanging oxygen from the lungs into the bloodstream and carbon dioxide from the bloodstream into the lungs, and exhaling air out of the body. The presentation also consists of a corresponding narration spoken in a human voice describing the processes being shown in the animation. Figure 1 shows frames from the animation along with the corresponding narration.

This is an example of a multimedia learning situation because the instructional message consists of words-in the form of narration-and pictures - in the form of animation (Mayer, 2001). During the past decade, researchers increasingly have been exploring ways of fostering meaningful learning in computer-based multimedia learning environments (Mayer, 2001; Rouet, Levonen, \& Biardeau, 2001; Sweller, 1999). The two most important paths toward fostering meaningful learning are (a) to design multimedia instructional messages in ways that reduce cognitive load (Mayer \& Moreno, 2003; Paas, Renkl, \& Sweller, 2003; Van Merrienboer, Kirschner, \& Kester, 2003), thus making more capacity available for deep cognitive processing during learning, and (b) to increase the learner's interest (Harp \& Mayer, 1998; Mayer, Sobko, \& Mautone, 2003; Moreno \& Mayer, 2000; Renninger, Hidi, \& Krapp, 1992), thus causing the learner to use the available capacity for deep processing during learning.

Examples of techniques to reduce cognitive load in computerbased multimedia presentations include eliminating extraneous words, sounds, and pictures (coherence principle), presenting

Richard E. Mayer, Sherry Fennell, Lindsay Farmer, and Julie Campbell, Department of Psychology, University of California, Santa Barbara.

Experiment 1 was conducted as part of a senior honors thesis by Sherry Fennell. Experiment 2 was conducted as part of a senior honors thesis by Lindsay Farmer. Experiment 3 was conducted by Julie Campbell. This research was supported by a grant from the Office of Naval Research.

Correspondence concerning this article should be addressed to Richard E. Mayer, Department of Psychology, University of California, Santa Barbara, CA 93106. E-mail: mayer@psych.ucsb.edu words as narration rather than as on-screen text (modality principle), placing on-screen text near rather than far from corresponding pictures (spatial contiguity principle), and presenting narrative simultaneously with corresponding animation rather than successively (temporal contiguity principle). Overall, design principles aimed at reducing cognitive load succeed when they free up limited cognitive capacity that was being used for extraneous processing and make it available for deep cognitive processing during learning. Design principles that reduce cognitive load in multimedia learning are based on a large and growing research base.

Examples of techniques to increase learner interest in computerbased multimedia presentations include using a human voice rather than a machine voice (voice principle) and using words in a conversational style rather than a formal style (personalization principle). Overall, design principles based on increasing interest succeed when they encourage learners to use their available cognitive capacity for active cognitive processing during learningthat is, to organize the presented material into coherent representations and integrate the pictorial and verbal representations with each other and with prior knowledge. In contrast to research on cognitive load, there is not yet a large research base concerning design principles that increase the learner's interest in multimedia learning.

The present set of studies contributes to the research base on design principles for increasing the learner's interest in multimedia learning, particularly, the personalization principle. In a previous set of studies, Moreno and Mayer (2000) found that students scored higher on a transfer test after receiving a narrated animation about lightning formation in which the words were in conversational style (i.e., using first and second person as well as comments directed at the learner) rather than in formal style (i.e., using third person and no comments directed at the learner). Moreno and Mayer (2000) also found that students scored higher on a transfer test after playing an educational science game containing narrated animation in which the words were in conversational style than when the words were in formal style. However, the treatment was somewhat heavy-handed, so the present studies examine whether personalization effects can be obtained with a more modest and focused personalization procedure-that is, changing "the" to 
"There are three phases in respiration--inhaling, exchanging, and exhaling."

1.

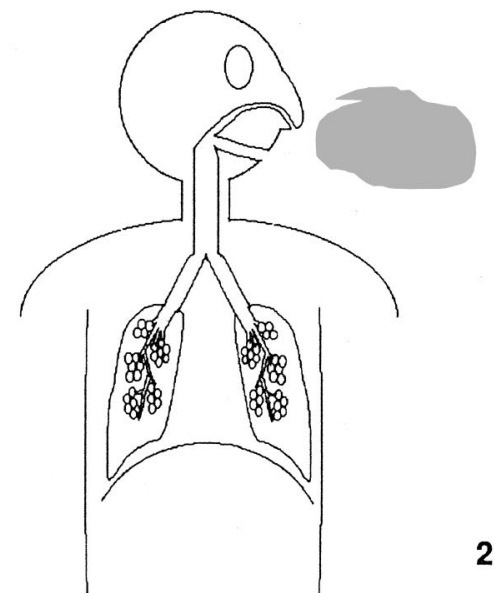

2.

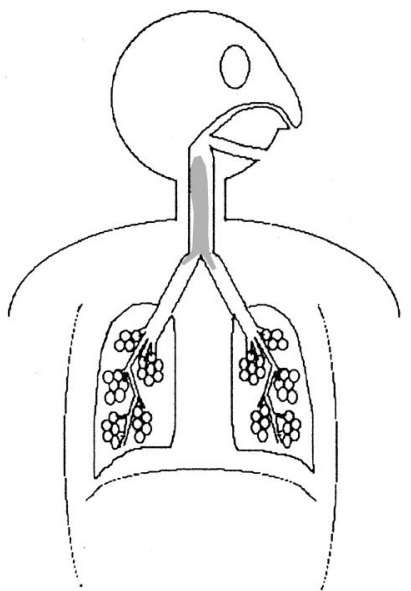

Inhaling

"During inhaling, the diaphragm moves down creating more space for the lungs, air enters through the nose or mouth, moves down through the throat and bronchial tubes to tiny air sacs in the lungs."
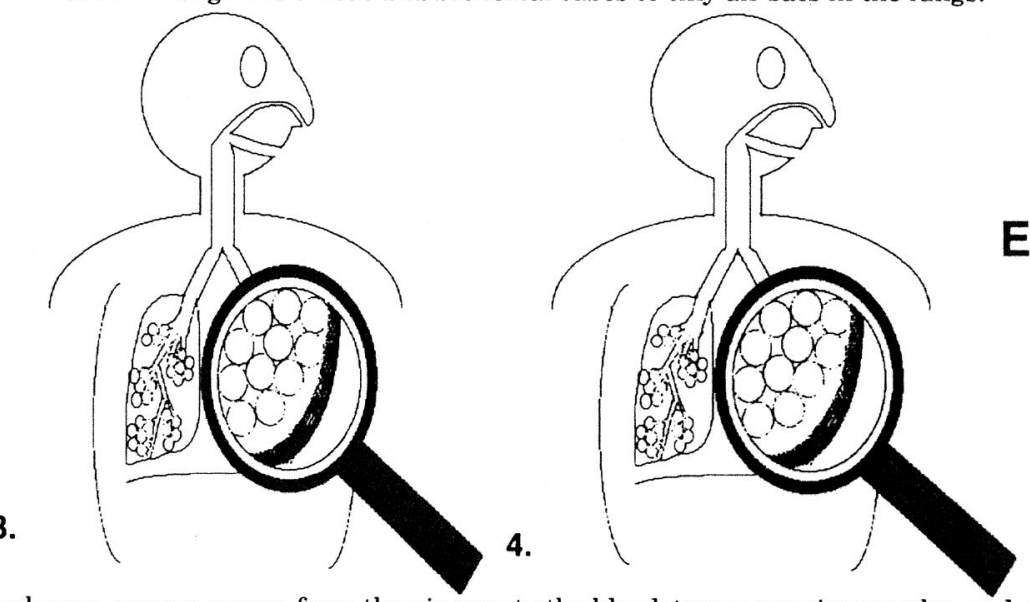

Exchanging

"During exchange, oxygen moves from the air sacs to the bloodstream running nearby, and carbon-dioxide moves from the bloodstream to the air sacs."

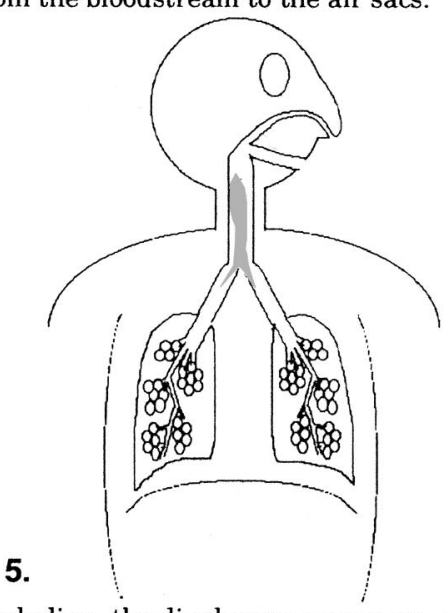

6.

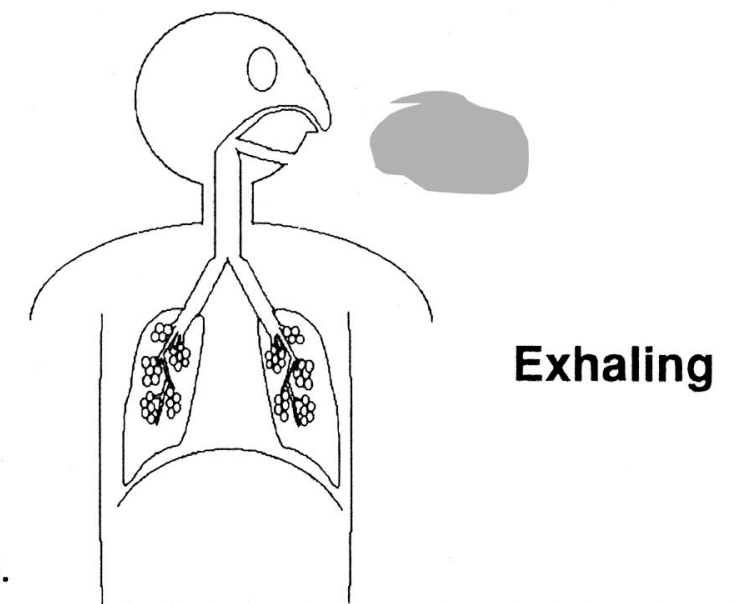

During exhaling, the diaphragm moves up , creating less room for the lungs, air travels through the bronchial tubes and throat to the nose and mouth, where it leaves the body."

Figure 1. Selected frames from a multimedia lesson on how the human respiratory system works, with corresponding narration. From "For Whom Is a Picture Worth a Thousand Words? Extensions of a Dual-Coding Theory of Multimedia Learning," by R. E. Mayer and V. K. Sims, 1994, Journal of Educational Psychology, 86, p. 398. Copyright 1994 by the American Psychological Association. 
"your" in 12 places throughout a narrated animation on how the respiratory system works.

The theoretical explanation for the effect of personalization is straightforward: Using the self as a reference point increases the learner's interest, which in turn encourages the learner to use available cognitive capacity for active cognitive processing of the incoming information during learning. The deeper processing results in more meaningful learning as indicated by better transfer test performance.

On the basis of this cognitive theory of multimedia learning, we predicted that the personalized group would score higher than the nonpersonalized group on transfer tests but not necessarily on retention tests. The rationale for this prediction is that both groups have adequate cognitive capacity for basic processing of the material and they both will pay attention to material and encode it; however, although both groups also have adequate cognitive capacity for deeper processing, students in the personalized group will be more likely to use that capacity for organizing the material and integrating it with prior knowledge because they are more interested in understanding the material. We refer to this as Prediction 1 , and we tested it in three experiments. We also predicted that students in the personalized group would be more interested in the material-as indicated by their ratings of how interesting the lesson was or their facial expressions during learning. We refer to this as Prediction 2, and we tested it in two experiments.

\section{Experiment 1}

In Experiment 1, we tested Prediction 1 by asking students to answer retention and transfer questions after viewing a personalized or nonpersonalized lesson on the human respiratory system.

\section{Method}

Participants and design. The participants were 62 college students recruited from the Psychology Subject Pool at the University of California, Santa Barbara. Twenty-nine participants served in the personalized group, and 33 served in the nonpersonalized group. The mean age was 18.5 years for the personalized group and 18.9 years for the nonpersonalized group; the personalized group contained $38 \%$ men, and the nonpersonalized group contained $33 \%$ men; the mean score (on a 12-point scale) on a survey of participants' experience with the tested information was 6.0 for the personalized group and 6.1 for the nonpersonalized group.

Materials and apparatus. The paper materials consisted of a participant questionnaire, a retention test sheet, and five transfer test sheets, with each printed on an $8.5 \times 11$ in. $(21.25 \times 27.5 \mathrm{~cm})$ sheet of paper. ${ }^{1}$ The participant questionnaire solicited basic demographic information, including the participant's age and gender, and included a two-part experience survey. The first part of the experience survey contained the instructions, "Please place a check mark next to the things you have done (check all that apply)." This was followed by 7 items: "taken a course in human anatomy/ physiology," "watched an educational program on how the respiratory system works," "taken a course in CPR or artificial respiration," "studied how the cardiovascular system and the respiratory system work together," "talked to a doctor about how the respiratory system works," "seen pictures of the structures of the lungs," and "used a computer-based lesson to understand how something works." The second part of the experience survey contained the instructions, "Please rate your knowledge of the human respiratory system (check one)." This was followed by "very low," "somewhat low," "average," "somewhat high," and "very high."

The retention test sheet had the following question printed at the top of the page: "Using what you learned in the lesson, please write an explana- tion of how the respiratory system works." The five transfer test sheets each had one of the following questions printed at the top of the page: "Suppose you are a scientist trying to improve the human respiratory system. How could you get more oxygen into the bloodstream faster?", "A researcher makes the claim that pollution causes heart disease. Explain why this would be true.", "Not enough oxygen is getting to the brain, and a person is about to faint. What could be wrong with the respiratory system?", "What mechanism in the body do you think allows you to breathe unconsciously, as you do when you are sleeping? (Hint: What causes the diaphragm to move down prior to inhaling and move up prior to exhaling?)", and "Please explain why oxygen transfers to the bloodstream and carbon dioxide transfers to the air sacs during the exchange phase of respiration."

The computer-based materials, developed using Director 6.0 (Macromedia, 1999), consisted of a personalized and a nonpersonalized version of a multimedia program explaining how the human respiratory system works. Both presentations were about $60 \mathrm{~s}$ long and consisted of the same animation depicting the process of inhaling air into the lungs, exchanging oxygen from the lungs into the bloodstream and carbon dioxide from the bloodstream into the lungs, and exhaling air out of the body (as shown in Figure 1). Both presentations also had an accompanying 100-word narration spoken in a male voice with a standard accent. The nonpersonalized version consisted of the following script (without the 12 bracketed words):

There are three phases in respiration: inhaling, exchanging, and exhaling. During inhaling, the [your] diaphragm moves down creating more space for the [your] lungs, air enters through the [your] nose or mouth, moves down through the [your] throat and bronchial tubes to tiny air sacs in the [your] lungs. During exchange, oxygen moves from the [your] air sacs to the bloodstream running nearby, and carbon dioxide moves from the bloodstream to the [your] air sacs. During exhaling, the [your] diaphragm moves up creating less room for the [your] lungs, air travels through the [your] bronchial tubes and throat to the [your] nose and mouth, where it leaves the [your] body.

The personalized version was identical except that in 12 places, the word, "the" was replaced with the word, "your" as is indicated by the 12 bracketed words. The only difference between the personalized and nonpersonalized presentations was that "the" (in the nonpersonalized version) was changed to "your" (in the personalized version) at 12 places in the narration. The paper materials and the nonpersonalized version of the multimedia lesson were adapted from Mayer and Sims (1994).

The apparatus consisted of four Macintosh G3 computer systems with 17-in. color monitors and Koss headphones. A stopwatch was also used.

Procedure. Participants were tested in groups of 1 to 4 and were randomly assigned to a treatment group. Each participant was seated in an individual cubicle, facing a computer screen. First, participants were given the participant questionnaire to complete at their own rate. Then, they were instructed that they would be viewing a short lesson on the human respiratory system and that afterward they would be asked some questions. The participants were instructed to put on the headphones and to use the mouse to click on the screen to start the program. When the participant clicked on the screen, the personalized or nonpersonalized lesson was presented, depending on the participant's treatment group. When all participants were finished viewing the lesson, the retention test sheet was passed out. Participants were instructed to write an answer to the question and to keep working until they were told to stop. The experimenter collected the

\footnotetext{
${ }^{1}$ In addition, in Experiments 1 and 3 two tests of spatial ability were administered; however, the data were not used because there were not enough participants to allow for further partitioning of the groups. An additional study was conducted that was similar to Experiment 1 but is not reported because of possible errors in data coding. As a replacement, Experiment 3 was conducted.
} 
retention test sheet after $5 \mathrm{~min}$. Then the first transfer test sheet was distributed, with instructions to "please write your answer to this question" and "keep working until you are told to stop." After $2.5 \mathrm{~min}$, the first transfer test sheet was collected. This process was repeated for each of the transfer test sheets, which were presented in the order listed in the Materials and apparatus section. After the final transfer test sheet, the participants were debriefed and excused. The experimenter followed APA standards for the ethical treatment of human participants.

\section{Results and Discussion}

Scoring. Each participant's retention test answer was scored by counting the number of idea units that had the same meaning as one of the 20 idea units in the narration: (1) There are three phases. (2) Inhaling, exhaling, and exchanging. (3) Diaphragm moves down. (4) Creating more space for the lungs (or allowing lungs to expand). (5) Air enters through nose or mouth. (6) Air moves down through the throat and bronchial tubes. (7) To tiny air sacs. (8) In the lungs. (9) Oxygen moves. (10) From the air sacs. (11) To the bloodstream. (12) Running nearby. (13) Carbon dioxide moves. (14) From the bloodstream. (15) To the air sacs. (16) The diaphragm moves up (or diaphragm contracts). (17) Creating less space for the lungs. (18) Air travels through the bronchial tubes and throat. (19) To the nose or mouth. (20) Where it leaves the body.

A transfer test score was computed for each participant by tallying the number of acceptable answers across each of the five transfer test sheets. Some acceptable answers for the first question ("Suppose you are a scientist trying to improve the human respiratory system. How could you get more oxygen into the bloodstream faster?") were to create more or larger air sacs or a more permeable bloodstream. Some acceptable answers to the second question ("A researcher makes the claim that pollution causes heart disease. Explain why this would be true.") were that less oxygen gets into the bloodstream, causing the heart to beat faster, or that polluted blood damages the heart as it circulates through the body. Some acceptable answers to the third question ("Not enough oxygen is getting to the brain, and a person is about to faint. What could be wrong with the respiratory system?") were that there is a blockage in the throat, that air sacs are congested, that there is a clog in the bloodstream, or that lungs are not expanding enough. An acceptable answer to the fourth question ("What mechanism in the body do you think allows you to breathe unconsciously, as you do when you are sleeping? Hint: What causes the diaphragm to move down prior to inhaling and move up prior to exhaling?") was that the brain receives a signal when the oxygen level gets low in the bloodstream. An acceptable answer for the final question ("Please explain why oxygen transfers to the bloodstream and carbon dioxide transfers to the air sacs during the exchange phase of respiration.") was that elements move from areas of higher concentration to areas of lower concentration. Overall, the maximum possible score was 20 for the retention test and 20 for the transfer test. The tests were scored by two raters; disagreements were resolved through consensus.

An experience score was computed for each participant by tallying the number of items checked on the checklist and by giving 1 to 5 points for the five possible answers on the rating scale (ranging from 1 for very low to 5 for very high).

Does personalization affect transfer performance? The top two rows of Table 1 show the mean scores and standard deviations
Table 1

Scores on Retention and Transfer Tests for Two Groups in Three Experiments

\begin{tabular}{|c|c|c|c|c|}
\hline \multirow[b]{2}{*}{ Group } & \multicolumn{2}{|c|}{ Retention } & \multicolumn{2}{|c|}{ Transfer } \\
\hline & $M$ & $S D$ & $M$ & $S D$ \\
\hline \multicolumn{5}{|l|}{ Experiment 1} \\
\hline Personalized & 11.3 & 3.3 & $7.1 *$ & 2.4 \\
\hline Nonpersonalized & 11.3 & 3.8 & 6.0 & 1.8 \\
\hline \multicolumn{5}{|l|}{ Experiment 2} \\
\hline Personalized & 8.2 & 3.2 & $7.8^{*}$ & 2.4 \\
\hline Nonpersonalized & 9.0 & 3.7 & 5.6 & 2.0 \\
\hline \multicolumn{5}{|l|}{ Experiment 3} \\
\hline Personalized & 11.1 & 3.9 & $6.2 *$ & 2.0 \\
\hline Nonpersonalized & 9.6 & 3.7 & 4.5 & 2.3 \\
\hline
\end{tabular}

Note. Asterisks indicate that the personalized group scored significantly higher than the nonpersonalized group.

for the two groups in Experiment 1 on retention and transfer. The mean score on the retention test was not significantly different for the two groups, $t(60)<1, p=n s$, yielding an effect size of -0.02 . The mean score on the transfer test was significantly greater for the personalized group than for the nonpersonalized group, $t(60)=$ $2.170, p=.034$, yielding an effect size of 0.65 . Overall, the results support Prediction 1; however, this experiment did not test Prediction 2 .

\section{Experiment 2}

Experiment 1 provided support for the idea that personalized instructional messages improve transfer performance (thus, supporting Prediction 1); however, Experiment 1 did not measure the learner's interest (or personal involvement) in learning (thus, not testing Prediction 2). In Experiment 2, we attempted to measure interest (or personal involvement) by counting the number of times the learner smiled while viewing the multimedia presentation.

\section{Method}

Participants and design. The participants were 27 college students recruited from the Psychology Subject Pool at the University of California, Santa Barbara. Fourteen participants served in the personalized group, and 13 served in the nonpersonalized group. The mean age was 18.8 years for the personalized group and 18.4 years for the nonpersonalized group; the personalized group contained $43 \%$ men, and the nonpersonalized group contained $38 \%$ men; the mean experience score (on a 12-point scale) was 6.8 for the personalized group and 6.9 for the nonpersonalized group.

Materials and apparatus. The materials were identical to those used in Experiment 1, except that the apparatus consisted of a single Macintosh ibook laptop computer without headphones and a Sony video camera on a tripod.

Procedure. The procedure was identical to that of Experiment 1, except that participants were tested individually, and their faces were video recorded while they watched the multimedia lesson.

\section{Results}

Scoring. Scoring of the retention and transfer tests was identical to that of Experiment 1. In addition, on the basis of the 
videotape, we tallied how many times each participant smiled during the multimedia lesson.

Does personalization affect transfer performance? The middle two rows of Table 1 show the mean scores and standard deviations for the two groups in Experiment 2 on retention and transfer. The mean score on the retention test was not significantly different for the two groups, $t(25)=0.594, p=.557$, yielding an effect size of -0.21 . The mean score on the transfer test was significantly greater for the personalized group than for the nonpersonalized group, $t(25)=2.516, p=.019$, yielding an effect size of 1.07. Overall, the results support Prediction 1.

Does personalization affect smiling during learning? None of the participants in either group showed any facial expressions during learning, suggesting that the measure was not sensitive. Experiment 2 seems to demonstrate that number of smiles is not a reasonable measure of interest. Overall, the experiment did not adequately test Prediction 2.

\section{Experiment 3}

In Experiment 2, facial expressions did not work as a measure of interest, so we explored another measure of interest in Experiment 3-asking students to rate how interesting the multimedia presentation was (i.e., interest rating). As a control, we also asked students to rate the difficulty of the multimedia presentation.

\section{Method}

Participants and design. The participants were 32 adults recruited from the Paid Subject Pool at the University of California, Santa Barbara. Each participant received $\$ 10$ for participating. Seventeen participants served in the personalized group, and 15 served in the nonpersonalized group. The mean age was 19.7 years for the personalized group and 20.7 years for the nonpersonalized group; the personalized group contained $18 \%$ men, and the nonpersonalized group contained $27 \%$ men; the mean experience score (on a 12-point scale) was 6.0 for the personalized group and 4.9 for the nonpersonalized group.

Materials and apparatus. The materials and apparatus were identical to those of Experiment 1, except that a difficulty rating sheet and interest rating sheet were added. ${ }^{2}$ The difficulty rating sheet contained the question, "How difficult was it for you to learn from this lesson? _ very easy, somewhat easy, _ slightly easy, _ neither easy nor difficult, slightly difficult, __ somewhat difficult, __ very difficult." The interest rating sheet contained the following question, "How interesting was this lesson? _ very interesting, __ somewhat interesting, __ slightly interesting, __ neither interesting or boring, __ slightly boring, __ somewhat boring, __ very boring."

Procedure. The procedure was identical to that of Experiment 1, except that after the multimedia lesson, participants completed the difficulty rating and interest rating at their own pace.

\section{Results}

Scoring. Scoring of the retention and transfer tests was identical to that of Experiment 1 . The difficulty rating was scored from 1 (very difficult) to 7 (very easy), and the interest rating was scored from 1 (very boring) to 7 (very interesting).

Does personalization affect transfer performance? The bottom two rows of Table 1 show the mean scores and standard deviations for the two groups in Experiment 3 on retention and transfer. The mean score on the retention test was not significantly different for the two groups, $t(30)=1.087, p=.286$, yielding an effect size of 0.37 . The mean score on the transfer test was significantly greater for the personalized group than for the nonpersonalized group, $t(30)=2.177, p=.037$, yielding an effect size of 0.72 . Overall, these results are consistent with Prediction 1.

Does personalization affect interest in learning? In contrast to Prediction 2, the mean score on the interest rating was not significantly greater for the personalized group $(M=5.1, S D=1.2)$ than for the nonpersonalized group $(M=4.5, S D=1.1), t(30)=$ $1.56, p=.129$, yielding an effect size of 0.55 . Consistent with Prediction 2, the mean difficulty rating of the personalized group $(M=5.9, S D=1.3)$ was not significantly different from the mean difficulty rating of the nonpersonalized group $(M=5.8, S D=$ $1.2), t(30)=0.324, p=.748$, effect size $=0.11$. Overall, there is not statistically significant evidence that personalization affects students' ratings of interest, but there is a trend in the predicted direction. The interest rating findings of Experiment 3 were more encouraging and suggestive than the findings related to smiling in Experiment 2, in that the interest measure yielded a larger difference in the predicted direction, this time approaching significance. Thus, it may be that the hypothesized role of interest as a mediator of the learning process is accurate, although it remains to be demonstrated. Overall, either the rating scale was not an entirely effective gauge for measuring interest, or personalization did not have the predicted positive effect on interest. Further research should include effective measures of interest and cognitive engagement.

\section{General Discussion}

\section{Theoretical Implications}

Making a seemingly minor change to 12 words (i.e., changing "the" to "your") in a multimedia lesson had a large effect on students' subsequent performance on tests of transfer-yielding effect sizes of $0.65,1.07$, and 0.72 across the three experiments, respectively. However, in each of the three experiments, these changes had no significant impact on retention of the presented words.

Why would this modest form of personalization have such a strong effect on transfer but not on retention? One answer to this question comes from the cognitive theory of multimedia learning: Personalization increases the learner's interest, increased interest causes the learner to exert more effort to engage in active cognitive processing during learning, and an increase in active cognitive processing during learning results in deeper learning, which is manifested in improved transfer performance. The level of effort that learners exert for the nonpersonalized version may be sufficient to promote basic processing in which the material is stored in long-term memory but without extensive organization and integration imposed on the material. Thus, with both personalized and nonpersonalized versions, students exerted sufficient cognitive processing to enable good performance on tests of retention. However, if personalized versions encourage additional constructive processing — such as organizing and integrating the material—-then

\footnotetext{
${ }^{2}$ In addition, in Experiment 3, following all other tests, students completed a 15-item scale rating the characteristics of the speaker, but the data were not used because the measure has been discredited.
} 
personalized versions should create their greatest effects on measures of transfer. This prediction-which we list as Prediction 1 -was strongly supported.

A complementary hypothesis is that students in the personalized group processed the material more deeply because they were more disposed to relate it to their prior knowledge. Thus, perhaps it is not an increase in the learner's overall interest that mediates depth of processing but rather the learner's sense that the topic of the discussion is personally relevant (including the activation of relevant prior knowledge). Although the personal relevance view and the interest view are not mutually exclusive, they emphasize somewhat different aspects of the mechanisms supposed to prime deeper processing. For example, according to the personal relevance view, future research should include detailed measures of the learner's relevant prior knowledge.

The missing links in our theoretical account, of course, concern measures of interest (or personal relevance) and measures of depth of processing during learning. In Experiment 2, we attempted to measure interest by recording students' facial expressions during learning, but this measure turned out to be insensitive. In Experiment 3 , we attempted to measure interest by asking students to rate their level of interest in the material they had just seen. According to the cognitive theory of multimedia learning, personalization should increase the students' interest in the material. We did not find statistically significant evidence for this prediction, so either our measurement instruments are somewhat defective or our hypothesis needs revision. Therefore, more focused research aimed at measuring interest and cognitive engagement would be helpful.

\section{Methodological Implications}

In these studies, we used multiple measures of learning outcomes-including both retention and transfer. Had we focused solely on measures of retention, which is often the case in instructional method studies, we would have concluded that the personalization treatment had no effect on learning. However, when we added measures of transfer-aimed at assessing meaningful learning outcomes - an entirely different pattern emerged. We are most interested in measures of transfer because transfer is generally recommended as a better measure of learner understanding than retention (Anderson et al., 2001). By using multiple measures, we were able to determine that although both groups remembered equivalent amounts of the presented text, the personalized group was better able to apply the material to new situations. Overall, this set of studies promotes the case for using multiple measures of learning outcomes, and in particular, for going beyond basic measures of amount remembered (Anderson et al., 2001).

\section{Practical Implications}

Concerning implications for practice, this research helps to establish a research base to support the personalization principle: In multimedia instructional messages, present the words in conversational style rather than in formal style. We suggest caution in applying this principle. Making extensive changes in the name of personalization can create seductive details (i.e., interesting but irrelevant words or pictures) that distract the learner, as demonstrated by Harp and Mayer $(1997,1998)$. Instead, we recommend a more subtle approach to creating personalization, such as the use of the word "your" (rather than "the") in the present experiments.

In a previous set of studies, Moreno and Mayer (2000) found that personalization improved transfer performance on an environmental science educational game and on learning from a multimedia lesson on lightning formation. Do the present experiments add anything new to the field? Given the somewhat unexpected effects reported by Moreno and Mayer, it was worthwhile to determine whether similar effects could be obtained in a new contextlearning about the human respiratory system. The overwhelming answer is yes, greatly adding to the credibility of the personalization effect. In addition, the personalization treatments used by Moreno and Mayer were somewhat heavy-handed and perhaps idiosyncratic, including adding a lot of new sentences to the instructional material. In the present study, we were interested in whether an extremely modest version of the personalization treatment-changing "the" to "your" in 12 places in a lesson-would also create a personalization effect. The overwhelming answer is yes. Overall, this set of experiments helps establish the robustness of the personalization effect and explores the conditions for the personalization effect (i.e., even a very modest change in the wording of the lesson is effective).

In summary, a major empirical contribution of this research is to establish that personalization can affect learner understanding. The personalization effect was strong and consistent over three experiments. A major theoretical contribution concerns identifying mechanisms underlying the personalization effect-such as the idea that the personalization primes deeper processing (including integration of the presented material with prior knowledge), which leads to superior transfer performance. Further work is needed, however, to develop direct measures of cognitive processing during learning (including, perhaps, brain activity recorded in functional magnetic resonance imaging studies), to directly test the idea that personalization primes deeper processing. In addition, further work is needed to determine whether an increase in the learner's interest or sense of personal relevance causes the hypothesized increase in cognitive processing during learning. Finally, a major practical contribution of this research is that a minor change in wording (i.e., using "your" instead of "the") can have a large practical effect on learning. The design implications are thatunder some circumstances-people may learn more deeply when the presented text is in conversational rather than formal style.

\section{References}

Anderson, L. W., Krathwohl, D. R., Airasian, P. W., Cruikshank, K. A., Mayer, R. E., Pintrich, P. R., et al. (2001). A taxonomy for learning, teaching, and assessing: A revision of Bloom's taxonomy of educational objectives. New York: Longman.

Harp, S. F., \& Mayer, R. E. (1997). The role of interest in learning from scientific text and illustrations: On the distinction between emotional interest and cognitive interest. Journal of Educational Psychology, 89, 92-102.

Harp, S. F., \& Mayer, R. E. (1998). How seductive details do their damage: A theory of cognitive interest in science learning. Journal of Educational Psychology, 90, 414-434.

Macromedia. (1999). Director 6.0 [Computer software]. San Francisco: Author.

Mayer, R. E. (2001). Multimedia learning. New York: Cambridge University Press. 
Mayer, R. E., \& Moreno, R. (2003). Nine ways to reduce cognitive load in multimedia learning. Educational Psychologist, 38, 43-52.

Mayer, R. E., \& Sims, V. K. (1994). For whom is a picture worth a thousand words? Extensions of a dual-coding theory of multimedia learning. Journal of Educational Psychology, 86, 389-401.

Mayer, R. E., Sobko, K., \& Mautone, P. D. (2003). Social cues in multimedia learning: Role of speaker's voice. Journal of Educational Psychology, 95, 419-425.

Moreno, R., \& Mayer, R. E. (2000). Engaging students in active learning: The case for personalized multimedia messages. Journal of Educational Psychology, 92, 724-733.

Paas, F., Renkl, A., \& Sweller, J. (2003). Cognitive load theory and instructional design: Recent developments. Educational Psychologist, $38,1-4$.
Renninger, K. A., Hidi, S., \& Krapp, A. (Eds.). (1992). The role of interest in learning and development. Hillsdale, NJ: Erlbaum.

Rouet, J., Levonen, J. J., \& Biardeau, A. (Eds.). (2001). Multimedia learning: Cognitive and instructional issues. Amsterdam: Elsevier.

Sweller, J. (1999). Instructional design in technical areas. Camberwell, Victoria, Australia: ACER Press.

Van Merrienboer, J. J. G., Kirschner, P. A., \& Kester, L. (2003). Taking the load off a learner's mind: Instructional design for complex learning. Educational Psychologist, 38, 5-14.

Received October 29, 2003 Revision received January 5, 2004 Accepted January 5, 2004

\section{Low Publication Prices for APA Members and Affiliates}

Keeping you up-to-date. All APA Fellows, Members, Associates, and Student Affiliates receive-as part of their annual dues-subscriptions to the American Psychologist and APA Monitor. High School Teacher and International Affiliates receive subscriptions to the APA Monitor, and they may subscribe to the American Psychologist at a significantly reduced rate. In addition, all Members and Student Affiliates are eligible for savings of up to $60 \%$ (plus a journal credit) on all other APA journals, as well as significant discounts on subscriptions from cooperating societies and publishers (e.g., the American Association for Counseling and Development, Academic Press, and Human Sciences Press).

Essential resources. APA members and affiliates receive special rates for purchases of APA books, including the Publication Manual of the American Psychological Association, and on dozens of new topical books each year.

Other benefits of membership. Membership in APA also provides eligibility for competitive insurance plans, continuing education programs, reduced APA convention fees, and specialty divisions.

More information. Write to American Psychological Association, Membership Services, 750 First Street, NE, Washington, DC 20002-4242. 